\title{
O.S.P.
}

L'orientation scolaire et professionnelle

$49 / 2$ | 2020

Genre, rapports sociaux de sexe et orientation

\section{Introduction : Genre, rapports sociaux de sexe et orientation}

Introduction: Gender relations and career choice and development

Jean-Philippe Gaudron

\section{(2) OpenEdition}

\section{Journals}

Édition électronique

URL : https://journals.openedition.org/osp/11912

DOI : 10.4000/osp.11912

ISSN : 2104-3795

Éditeur

Institut national d'étude du travail et d'orientation professionnelle (INETOP)

Édition imprimée

Date de publication : 20 juin 2020

Pagination : 193-198

ISSN : 0249-6739

Référence électronique

Jean-Philippe Gaudron, «Introduction: Genre, rapports sociaux de sexe et orientation », L'orientation scolaire et professionnelle [En ligne], 49/2 | 2020, mis en ligne le 20 juin 2020, consulté le 20 juin 2022. URL : http://journals.openedition.org/osp/11912 ; DOI : https://doi.org/10.4000/osp.11912

Ce document a été généré automatiquement le 20 juin 2022.

(C) Tous droits réservés 


\title{
Introduction : Genre, rapports sociaux de sexe et orientation
}

\author{
Introduction: Gender relations and career choice and development
}

\author{
Jean-Philippe Gaudron
}

1 En lançant un appel à contribution Genre, rapports sociaux de sexe et orientation, il s'agissait pour la revue l'Orientation Scolaire et Professionnelle de rendre compte des recherches menées à l'aide de ces paradigmes sociologiques sur les différences entre les filles et les garçons, les femmes et les hommes, dans les choix scolaires et professionnels ainsi que le développement de carrière. Certes, des psychologues ont su rapidement invoquer et utiliser le concept de rapports sociaux de sexe pour rendre compte justement de ces différences et surtout des inégalités entre les deux sexes en matière d'orientation (Bosscher \& Durand-Delvigne, 2002 ; Faurie \& Costalat-Founeau, 2016 ; Mieyaa et al., 2012 ; Molinier, 2002). Et la revue étant pluridisciplinaire, quelques sociologues (Devineau, 2013; Schuft \& Ciavaldini-Cartaut, 2016), ainsi que des chercheures en Sciences de l'éducation (Mikanga \& Joulain, 2018), ont aussi présenté à notre lectorat des études sur cette thématique. Au final, l'idée de ce numéro a été d'attirer et de mettre en valeur ce type d'approches sociologiques, d'en montrer l'actualité et d'en souligner tout l'intérêt pour la recherche et la pratique, pour les disciplines préoccupées par l'orientation, et en particulier la psychologie.

La sociologie a proposé plusieurs paradigmes et concepts pour comprendre et expliquer ces différences entre les femmes et les hommes qui reflètent selon elle, leurs positions dans la hiérarchie sociale, et parmi ceux-ci, les rapports sociaux de sexe (RSS). Qu'estce qu'un rapport social ? Dans le Dictionnaire Critique du Féminisme (Hirata et al., 2004), Kergoat définit le rapport social comme « une tension qui traverse le champ social et qui érige certains phénomènes sociaux en enjeux autour desquels se constituent des groupes sociaux aux intérêts antagonistes" (p. 34). Il est dès lors question de RSS lorsqu'il s'agit du groupe social femmes et du groupe social hommes, et parmi les enjeux autour desquels ces deux groupes sont en tensions permanentes, il y a la division sexuelle du travail. La division sexuelle du travail a deux principes organisateurs (Kergoat, 2010) : le principe de séparation et le principe hiérarchique. Le 
premier principe renvoie à la division horizontale du travail, la ségrégation des travaux, des métiers ou des filières de formation. Le second principe renvoie à la division verticale du travail : les travaux, métiers ou filières de formation des hommes ont plus de valeur que ceux des femmes. Enfin, les RSS sont caractérisés par plusieurs dimensions (Hirata et al., 2004, p. 40) : «La relation entre les groupes est antagonique ; les différences constatées entre les pratiques des hommes et des femmes sont des construits sociaux et ne relèvent pas d'une causalité biologique ; ce construit social a une base matérielle et pas seulement idéologique; ces rapports sociaux reposent d'abord et avant tout sur un rapport hiérarchique entre les sexes ".

3 Telle que définie, cette conceptualisation des RSS présente de nombreuses similitudes avec le concept de genre dans son acceptation sociopolitique. Ainsi, pour Béréni, Chauvin, Jaunait et Révillard (2008), le genre est un «système de bicatégorisation hiérarchisée entre les sexes (hommes/femmes) et entre les valeurs et représentations qui leur sont associées (masculin/féminin)»(p. 7). Cette définition permet, selon ces auteurees, de rendre compte de quatre dimensions : les différences entre les hommes et les femmes ne sont pas le produit d'un déterminisme biologique, mais d'une construction sociale; les caractéristiques associées à chaque sexe sont socialement construites dans une relation d'opposition; les relations sociales entre les sexes sont un rapport de pouvoir ; le genre est à l'intersection d'autres rapports de pouvoir.

4 Pour Pfefferkorn (2012) le terme RSS présente néanmoins certains avantages sur le terme de genre: il met l'accent sur les dimensions matérielles de l'oppression sans négliger les dimensions symboliques ; il met en exergue les conflits autour du travail ; il permet d'articuler entre eux les différents rapports sociaux qui traversent la société : rapport social de sexe, rapport social de classe, rapport social de racisation; il permet enfin de penser le changement: "il s'agit de penser en même temps comment les sujets, hommes et femmes, suivant leur place dans les rapports sociaux sont contraints structurellement et façonnés au niveau et dans l'espace où ils se trouvent; et comment ces mêmes sujets, par leur activité, individuelle et collective, par leurs actions réciproques, peuvent construire des marges de liberté et d'action leur permettant de déplacer ces rapport sociaux » (p. 108).

5 Si ce dernier avantage présentera une forte résonnance chez nombre de psychologues, que faire alors de ces déterminants sociologiques que sont les classes de sexe et les classes sociales, et dont on connaît l'importance fondamentale dans les procédures institutionnelles d'affectation, les conduites individuelles et les pratiques professionnelles en orientation tout au long de la vie (Heppner \& Jung, 2013). Ou plus précisément, pour paraphraser la question posée par Liu et ses collaborateurs (Liu et al., 2004) à propos du concept de classe sociale : comment, nous autres psychologues, pouvons-nous comprendre et utiliser «psychologiquement» ce concept de rapports sociaux de sexe?

6 Les six études rassemblées dans ce numéro spécial proposent, sinon des réponses à cette question, du moins des éclairages différents et variés sur les apports et intérêts d'une lecture en termes de rapports sociaux de sexe des choix scolaires et professionnels et du déroulement de carrière. Elles ont été menées par des chercheure's en sociologie et en psychologie, travaillant dans les laboratoires au Canada et en France: l'Orientation Scolaire et Professionnelle est une revue internationale et pluridisciplinaire. Les deux premiers articles portent sur la formation initiale. Prisca Kergoat s'intéresse à l'apprentissage ou plus précisément aux grandes 
absentes de ce dispositif : les filles. L'enquête MADAA sur la mesure des discriminations à l'entrée de l'apprentissage que présente cette contribution, articule deux méthodologies, quantitative et qualitative. Sur la base des statistiques de répartition, l'auteure formule et teste à l'aide d'entretiens ethnographiques l'hypothèse que ces distributions différentes entre les sexes trouvent leur origine non pas dans les choix des filles et des garçons, mais dans la sélection à l'entrée des dispositifs de formation. Prisca Kergoat montre également comment la notion de consubstantialité permet de repérer les relations d'interdépendance entre les différents rapports sociaux.

7 Pierre Doray, Alexandre Lépine et Jaunathan Bilodeau se penchent sur la poursuite des études des femmes qui ont choisi de suivre des formations majoritairement composées d'hommes au Québec. Leur étude comporte également deux volets : l'un quantitatif et, à partir des données du ministère de l'Éducation et de l'Enseignement supérieur du Québec, les auteurs proposent plusieurs indicateurs permettant d'identifier les facteurs en jeu dans les abandons et changements de formations, plus nombreux chez les femmes que chez les hommes; l'autre qualitatif et, sur le base d'entretien de recueil d'expériences vécues par des femmes qui ont suivi ce type de formation, les chercheurs proposent des analyses qui montrent comment se manifestent ou s'actualisent les rapports sociaux de sexe.

8 Les deux articles suivants portent sur le déroulement de carrière d'hommes qui travaillent dans des métiers dits « de femmes » et inversement, de femmes qui exercent des fonctions dites «d'hommes ». Sophie Devineau, François Féliu, Sandrine Valentin et Clémence Bernard s'intéressent à ces hommes qui ont choisi de travailler dans les métiers de la petite enfance, dans des crèches, des écoles maternelles ou comme assistants maternels. Soixante-quinze hommes ont ainsi participé à des entretiens livrant des parcours biographiques riches et variés à partir desquels les chercheureess se proposent de saisir les multiples éléments ayant conduit ces hommes à s'orienter vers ces métiers du care. Puis les auteur'e's vont tenter d'identifier des mécanismes de réassignations de genre, et leurs liens avec les rapports sociaux de sexe, les stéréotypes de sexe et le sexisme.

Émilie Giguère, Louise St-Arnaud et Karine Bilodeau se proposent d'étudier les parcours scolaires et les parcours d'insertion socioprofessionnelles de cinquante et une femmes cadres canadiennes. Les entretiens menés sont analysés à l'aide des catégories conceptualisantes et mis en rapport avec deux perspectives théoriques: la sociologie des rapports sociaux de sexe et la théorie du travail humain en psychodynamique du travail. Les données montrent comment s'actualisent les rapports sociaux de sexe, dès le parcours scolaire, lors de l'insertion professionnelles, dans les relations avec les collègues ou pour le travail domestique; et quelles stratégies d'adaptation individuelle ces femmes mettent en œuvre pour y faire face.

Le cinquième article offre un éclairage bien en amont de l'orientation. Fabienne Montmasson-Michel s'intéresse à la socialisation langagière des très jeunes enfants et les influences conjuguées de quatre instances socialisatrices : l'école, le groupe de pairs, les familles et l'industrie culturelle. Cette recherche aussi donne l'occasion de présenter une enquête ethnographique menées auprès d'enfants scolarisés dans des écoles maternelles, leur famille et les acteurs et actrices de l'Éducation nationale (enseignant·e·s, agents territoriaux...). Les résultats et les analyses proposées montrent comment, et de façon très précoce, les filles et les garçons - en intériorisant deux ordres sociaux, ceux des rapports sociaux de sexe et de classe - construisent des façons 
différentes de s'identifier, de développer des rapports aux autres, des rapports au savoir..., qui pourront se traduire plus tard par des choix d'orientation différents.

Enfin, dans une dernière contribution, Jean-Philippe Gaudron présente une étude quantitative sur les croyances à propos des différences entre les filles et les garçons, les femmes et les hommes quant à leurs choix scolaires et professionnels, leur déroulement de carrière et l'articulation travail/famille. L'article décrit le développement et la validation psychométrique d'une échelle qui confronte trois croyances, trois visions du monde : l'essentialisme psychologique, le constructionnisme social et le féminisme matérialiste. L'auteur propose de nommer ce questionnaire "l'échelle de conscience des rapports sociaux de sexe ", en référence à Liu et ses travaux sur la conscience des classes sociales.

\section{BIBLIOGRAPHIE}

Bereni, L., Chauvin, S., Jaunait, A., \& Révillard, A. (2008). Introduction aux Gender Studies : Manuel des études sur le genre. De Boeck.

Bosscher, S. de, \& Durand-Delvigne, A. (2002). Asymétrie sociale et asymétrie cognitive : Étude de l'opérativité des catégories de sexe. L'orientation scolaire et professionnelle, 31(1), 57-74. https:// doi.org/10.4000/osp.4848

Devineau, S. (2013). Devenir enseignant·ess dans le public : Le sens d'une orientation professionnelle très féminisée. L'orientation scolaire et professionnelle, 42(2), 245-272. https:// doi.org/10.4000/osp.4127

Faurie, I., \& Costalat-Founeau, A.-M. (2016). Sentiments d'efficacité personnelle et dynamique capacitaire dans les choix d'orientation atypique. L'orientation scolaire et professionnelle, 45(2). https://doi.org/10.4000/osp.4971

Heppner, M. J., \& Jung, A.-K. (2013). Gender and social class: Powerful predictors of a life journey. In W. B. Walsh, M. L. Savickas, \& P. J. Hartung (Éds.), Handbook of vocational psychology: Theory, research, and practice ( ${ }^{\text {th }}$ ed., pp. 81-102). Routledge/Taylor \& Francis Group.

Hirata, H., Laborie, F., Le Doaré, H., \& Laborie, F. (2004). Dictionnaire critique du féminisme ( $2^{\mathrm{e}}$ éd. augmentée). Presses Universitaires de France.

Kergoat, D. (2010). Le rapport social de sexe de la reproduction des rapports sociaux à leur subversion. In A. Bidet-Mordrel (Ed.), Les rapports sociaux de sexe (pp. 60-75). Actuel Marx Confrontations, PUF.

Liu, W. M., Ali, S. R., Soleck, G., Hopps, J., Dunston, K., \& Pickett, T. Jr. (2004). Using Social Class in Counseling Psychology Research. Journal of Counseling Psychology, 51(1), 3-18. https://doi.org/ 10.1037/0022-0167.51.1.3

Mieyaa, Y., Rouyer, V., \& Blanc, A. le. (2012). La socialisation de genre et l'émergence des inégalités à l'école maternelle : Le rôle de l'identité sexuée dans l'expérience scolaire des filles et des garçons. L'orientation scolaire et professionnelle, 41(1), 57-75. https://doi.org/10.4000/osp.3680 
Mikanga, E., \& Joulain, M. (2018). Déterminants scolaires et professionnels des femmes et des hommes s'orientant vers un métier dit « féminin », le cas des aides médico-psychologiques (AMP). L'orientation scolaire et professionnelle, 47(4), 659-681. https://doi.org/10.4000/osp.8919

Molinier, P. (2002). Féminité sociale et construction de l'identité sexuelle : Perspectives théoriques et cliniques en psychodynamique du travail. L'orientation scolaire et professionnelle, 31(4), 565-580. https://doi.org/10.4000/osp.3438

Pfefferkorn, R. (2012). Genre et rapports sociaux de sexe. Éditions Page deux.

Schuft, L., \& Ciavaldini-Cartaut, S. (2016). Genre et représentations de l'autorité chez les enseignant·e's débutant·e's exerçant en lycée professionnel. L'orientation scolaire et professionnelle, 45(3), 331-355. https://doi.org/10.4000/osp.5048

\section{AUTEUR}

\section{JEAN-PHILIPPE GAUDRON}

Professeur en psychologie, UMR Éducation, Formation, Travail, Savoirs (EFTS) (MA 122),

Université Toulouse 2 Jean Jaurès. Courriel : jean-philippe.gaudron@univ-tlse2.fr 